\title{
Superfluidity of two-dimensional excitons in flat and harmonic traps
}

\author{
Yu.E. Lozovik ${ }^{a}$, I.L. Kurbakov ${ }^{a}$, and Magnus Willander ${ }^{b}$ \\ a Institute of Spectroscopy, Russian Academy of Sciences, 142190 Troitsk, Moscow region, Russia \\ ${ }^{b}$ Institute of Science and Technology (ITN), Linköping University, SE-581 83, Linköping, Täppan 6177, Sweden
}

(Dated: October 30, 2018)

\begin{abstract}
Superfluid exciton density and superfluid transition (crossover) temperature are calculated for 2D excitons in large-size flat and harmonic traps. A generalized local density approximation for the Kosterlitz-Thouless theory is developed.
\end{abstract}

PACS numbers: 71.35.Lk, 73.21.Fg, 73.63.Hs

\section{INTRODUCTION}

Two-dimensional (2D) indirect exciton systems in coupled quantum wells (CQWs) [1] (or in single QW in strong normal electric field [2]), in which electrons and holes are spatially separated, at low temperatures can possess condensation and superfluidity [1, 2, 3, 4, 4, 5, 6] (or crystallization in some region of phase diagram [6, 7, 8$]$ ), persistent electric currents and quasi-Josephson effects 9|, as well as interesting coherent optical phenomena [10]. There is a tremendous progress now in the observation of collective exciton properties in CQWs (see reviews [11, 12, 13, 14]).

Due to the divergence of phase fluctuations, Bose condensation of excitons (possible in 3D systems 15]) in extended 2D exciton systems at $T \neq 0$ is impossible [16]. But at low temperatures the system can possess quasilong (power-law) off-diagonal order [17] and superfluidity, which are destroyed by vortex pair dissociation and free vortex formation at Kosterlitz-Thouless (KT) temperature [18, 19, 20]. But Bose condensation is possible in finite 2D systems 21]. Besides, the trapping can increase the exciton density at fixed laser pumping, which leads to the growth of Bose condensation temperature. Several types of traps were proposed and experimentally realized: (i) "natural" traps created by (one-monolayer) well width fluctuations [22, 23, 24]; and regular, controlled traps such as (ii) laser-induced traps formed inside the annulus of laser pump [25], (iii) traps formed by an inhomogeneous deformation created by a needle stressing on the sample [26, 27], (iv) mesa created by engineering width of layers or the barrier in CQWs, and (v) different kinds of electrostatic traps [12, 28, 29, 30]. The interesting optical properties of exciton collective state are observed in luminescence measurements 22, 23, 24, 25, 30, 31, 32, 33] (see also [11, 12, 13, 14]).

In connection with the discussion above essential problems arose concerning the nature of exciton collective state in 2D traps: (i) How the phase fluctuations and vortices manifest itself in sufficiently large but finite traps? (ii) How the type of the confining potential influences on the superfluid density spatial distribution and its evolution with the temperature? These problems the present paper are mainly addressed to.

We describe quantitatively the superfluidity of $2 \mathrm{D}$
CQW excitons in 2D large-size flat trap by means of KT theory. For the investigation of the exciton superfluidity in 2D large-size harmonic trap we employ the local density approximation [34] and develop more correct generalized local density approximation for KT theory.

\section{SUPERFLUIDITY OF 2D EXCITONS IN A LARGE-SIZE HARMONIC TRAP: THE LOCAL DENSITY APPROXIMATION}

As the first step for study of 2D CQW exciton superfluidity in a large-size harmonic trap we use the local density approximation (LDA). LDA is valid if confining potential of the trap varies sufficiently slowly on all microscopical characteristic scales of the problem, particularly, related to phonon and vortex excitations in the exciton system. Therefore, in LDA exciton superfluid properties near the trap point $\mathbf{R}$ coincides with properties of the homogeneous infinite superfluid with total density $n_{t}^{\infty}$ being equal to the (total) exciton density $n_{t}(\mathbf{R})$ in the trap in the point $\mathbf{R}$.

A homogeneous infinite exciton system at fixed temperature $T$ is a superfluid at high total densities $n_{t}^{\infty}$ but it is a normal fluid at low $n_{t}^{\infty}$. As total exciton density $n_{t}^{\infty}$, when decreasing, goes through a critical one $\tilde{n}_{t}^{\infty}$ corresponding to exciton temperature $T$ the superfluid KT transition is occured in the system and superfluid exciton density $n_{s}^{\infty}$ vanishes by the universal jump $\tilde{n}_{s}^{\infty}=2 m T / \pi \hbar^{2}$ [35] ( $m$ is the exciton mass).

So, the trapped excitons in LDA are superfluid (normal) ones at the trap point $\mathbf{R}$ at which total exciton density $n_{t}(\mathbf{R})$ is greater (less) than the critical one $\tilde{n}_{t}^{\infty}$, corresponding to exciton temperature $T$. Thus, in LDA at the sufficiently low temperatures at which the critical total exciton density $\tilde{n}_{t}^{\infty}$ is smaller than maximal one $n_{t}^{m}=n_{t}(0)$ the exciton system in a symmetrical harmonic trap is divided into a superfluid circle $R<R_{s}$ of a radius $R_{s}$ and normal ring $R_{s}<R<\bar{L}$ surrounding the circle ( $\bar{L}$ is the Thomas-Fermi (TF) radius). In the superfluid circle the total exciton density $n_{t}(R)$ exceeds a critical value $\tilde{n}_{t}^{\infty}$ corresponding to exciton temperature $T$. But the superfluid density $n_{s}(R)$ is proved (see details in [19, 35]) to be higher than the universal jump $\tilde{n}_{s}^{\infty}$ for this temperature. In opposite, in the normal ring total exciton density $n_{t}(R)$ is smaller than $\tilde{n}_{t}^{\infty}$ and superfluid 
density is equal to zero $n_{s}(R) \equiv 0$. In the circumference $R=R_{s}$ which is the boundary line of the superfluid circle and normal ring the local KT transition takes place on which the onset of pair vortex dissotiation and free vortex formation occurs. At the circumference $R=R_{s}$ the superfluid density jumps from $\tilde{n}_{s}^{\infty}$ to zero.

As the temperature grows critical total exciton density $\tilde{n}_{t}^{\infty}$ increases. So, the superfluid circle shrinkes and the normal ring thickens. At the temperature $\bar{T}_{c}^{\infty}$, at which the critical total density becomes equal to maximal total density in the whole trap $n_{t}^{m}=n_{t}(0)$, the superfluid circle disappears and the trapped exciton system undergoes a global transition to the normal state.

\section{SUPERFLUIDITY OF 2D EXCITONS IN A LARGE-SIZE FLAT TRAP}

In $2 \mathrm{D}$ homogeneous (flat) trap of sufficiently large size $L$ the true, vortex-renormalized (VR), superfluid exciton density $n_{s}$ takes into account the renormalization ("screening") by all vortex pairs in the system, i.e., by vortex pairs with the separation less than $L$. So, VR superfluid density $n_{s}$ is expressed through "local", vortexunrenormalized (VU), superfluid density $n_{l}$ as follows

$$
n_{s}=n_{l} / \epsilon(L)
$$

where $\epsilon(r)$ is vortex pair dielectric function [19, 36, 37]. In superfluid phase where there is a rare vortex pair gas the quantity $\epsilon(L)$ according to KT model [19, 36] obeys the equation

$$
\frac{d \epsilon(x)}{d x}=C(a) e^{4 x-a \int_{0}^{x} d x^{\prime} / \epsilon\left(x^{\prime}\right)}
$$

with the boundary condition

$$
\epsilon(0)=1 \text {. }
$$

Here $x=\ln \left(L / r_{0}\right), r_{0}$ is vortex core diameter (in CQWs $r_{0}$ is of order of the distance between excitons [38]),

$$
a=2 \pi \hbar^{2} n_{l} / m T,
$$

and $C(a)$ is a (nonuniversal) constant depending on microscopical exciton superfluid properties; in 2D $X-Y$ model it is equal to $C(a)=\pi^{2} a e^{-\pi a / 2}$.

According to our numerical analysis of Eqs. (11)-(3), at low temperatures $T$ (i.e., at large $a$; see Eq. (4)) VR superfluid density $n_{s}$ is close to VU one $n_{l}$. As $T$ grows the ratio $n_{s} / n_{l}$ decreases. At a critical temperature $T_{c}^{L}$ in sufficiently large traps $\left(\ln \left(L / r_{0}\right) \gg 1\right)$ there is the crossover where rather sharp disappearance of the VR superfluid density takes place. The disappearance corresponds to the onset of pair vortex dissociation and free vortex formation [19], superfluid state being destroyed.

Numerical integrating Eqs. (2), (3) yield the following expression for the superfluid crossover temperature for CQW excitons in 2D flat trap of size $L$ :

$$
T_{c}^{L}=\frac{\pi \hbar^{2} n_{l}\left(T_{c}^{L}\right)}{2 m \tilde{\epsilon}^{L}},
$$

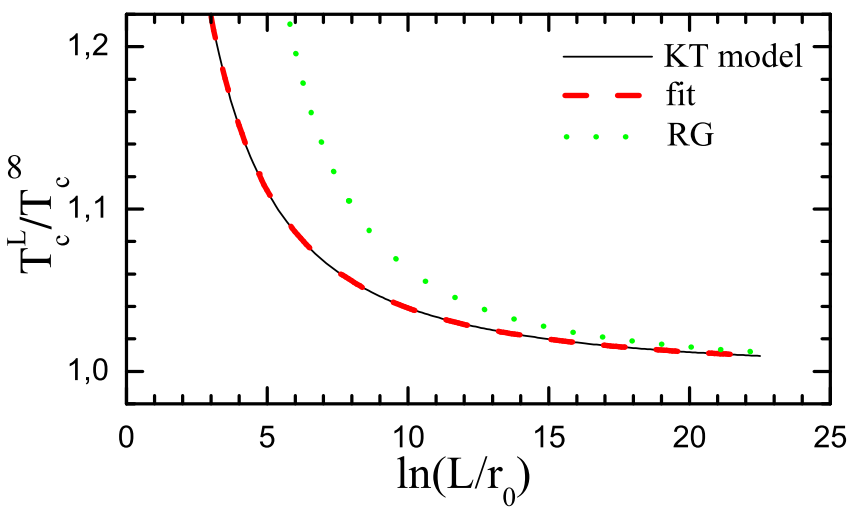

FIG. 1: The dependence of the superfluid crossover temperature $T_{c}^{L}$ of $2 \mathrm{D}$ CQW excitons in the flat trap on its size $L$ $\left(\left.T_{c}^{\infty} \equiv T_{c}^{L}\right|_{L=\infty}\right)$. Thin solid line is the numerical intergation of the equation for vortex pair dielectric function $\epsilon(L)$ in $\mathrm{KT}$ model, dash line is the fit $\tilde{\epsilon}^{\infty} / \tilde{\epsilon}^{L}$ (according to Eq. (6)), and dot line is the Kosterlitz's renormalization group calculation.

where $n_{l}\left(T_{c}^{L}\right)$ is the VU superfluid density of $2 \mathrm{D}$ excitons at the crossover (at $T=T_{c}^{L}$ ) and the quantity $\tilde{\epsilon}^{L}$ is calculated numerically. The fit

$$
\tilde{\epsilon}^{L}=\left(1-\frac{\pi^{2} b^{2}}{\left(\ln \left(L / r_{0}\right)+\Delta\right)^{2}}\right) \tilde{\epsilon}^{\infty}
$$

coincides excellently with the numerical calculation at large $L$ (see below and Fig. 1); in Eq. (6) $\tilde{\epsilon}^{\infty}$ is the critical vortex pair dielectric constant in $2 \mathrm{D}$ infinite superfluid at $\mathrm{KT}$ transition, $b$ and $\Delta$ are nonuniversal quantities which, as $\tilde{\epsilon}^{\infty}$, depend on microscopical exciton system properties. In $2 \mathrm{D} X-Y$ model we obtain $\tilde{\epsilon}^{\infty}=1.134(9)$, $\Delta=2 .(93)+\tilde{\Delta}$ and $b=0.8(00)$. The quantity $\tilde{\Delta}=\tilde{\Delta}(L)$ determines the correctness of KT model, so, within KT model one must assume that $\tilde{\Delta}=0$. The numerical calculation of the width of superfluid crossover region, in which vortex pairs should begin to dissociate, yields the following estimation $e^{\tilde{\Delta}} \sim 1$.

From Eqs. (5), (6) we conclude that the approach used takes into account the second order on the logarithmically small quantity $1 /\left(\ln \left(L / r_{0}\right)+\Delta\right)$. Thus, our calculation is correct in the logarithmic approximation $\left(\ln \left(L / r_{0}\right) \gg 1\right)$, i.e., for sufficiently large traps.

For the halfwidth of superfluid crossover region we obtain (see Eqs. (5) and (66)

$$
\Delta T_{c}^{L} \sim T_{c}^{L} \frac{\pi^{2} b^{2}}{\left(\ln \left(L / r_{0}\right)+\Delta\right)^{3}} .
$$

Thus, the model adopted does not take into account effects in the crossover region (because $\Delta T_{c}^{L}$ depends on the third order of $\left.\ln \left(L / r_{0}\right) \gg 1\right)$. In the crossover temperature region $\Delta T_{c}^{L}$ the number of free vortices inside the trap area $L^{2}$ increases by $e$ times, whereas at $T=T_{c}^{L}$ there is (in average) of order of one free vortex.

Note that the result (7) for 2D differs essentially from the case of 3D Bose condensed systems where $\Delta T_{c}^{L}$ depends on a power of $L$. 


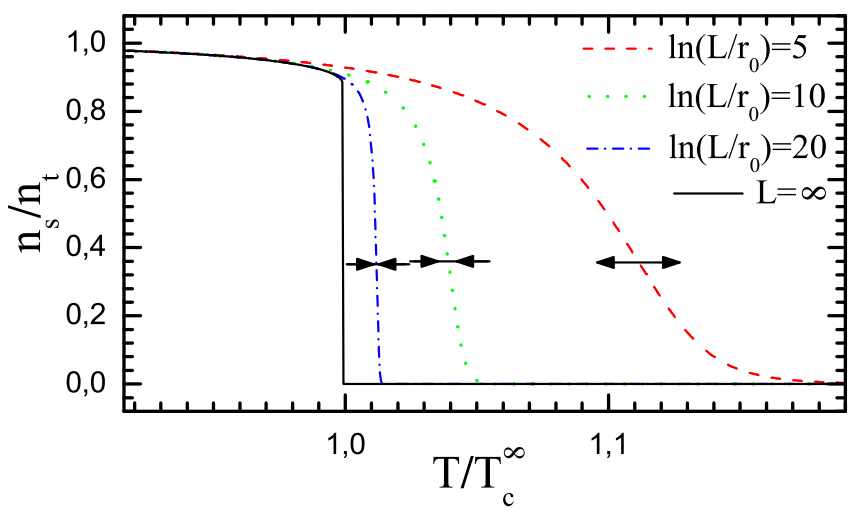

FIG. 2: The dependence of the superfluid density of 2D CQW excitons in the flat trap of different size $L$ on the ratio of exciton temperature $T$ to the superfluid transition temperature $T_{c}^{\infty}$ of $2 \mathrm{D}$ infinite systems. The regions of the superfluid crossovers on which the superfluid density universal jump is smeared out are shown by arrows.

For the completeness of the calculation of VR superfluid density $n_{s}$ and superfluid crossover temperature $T_{c}^{L}$ in the flat trap one must calculate the dependence of VU superfluid density $n_{l}$ on temperature $T$ and total density $n_{t}$ (see Eqs. (11), (44) and (50). This calculation is realized in Refs. [38, 39]. In this Letter we make a simplification. Namely, for spatially indirect CQW excitons the strong dipole-dipole repelling results in a small thermal depletion of their $V U$ superfluid density (not the condensate) throughout the superfluid phase (at $T<T_{c}^{L}$ ) [38]. In a sufficiently large flat trap in which the condition $\tilde{\epsilon}^{L}>\tilde{\epsilon}^{\infty} / 1.2$ is hold [40] VU superfluid density $n_{l}$ thoughout the superfluid phase obeys to the inequality $n_{t}>n_{l}>n^{*}$, where $n^{*} \equiv n_{l}\left(T_{c}^{L}\right)=(0.93 \div 0.98) n_{t}$ for GaAs CQWs studied experimentally in Refs. 22, 23, 24, 25, 26, 30, 32, 33]) and $n^{*} \approx 0.9 n_{t}$ in Refs. [27, 31]. So, $n_{l}$ is close to the total exciton density $n_{t}$ (for pure CQWs) [38]. Thus, for spatially indirect CQW excitons we can assume VU superfluid exciton density $n_{l}$ to be approximately equal to total one $n_{t}$ throughout the superfluid phase

$$
n_{l} \approx n_{t} \quad\left(T<T_{c}^{L}\right) .
$$

This permits not only to simplify Eqs. (10), (4) and (5)

$$
\begin{gathered}
n_{s}=\frac{n_{t}}{\epsilon(L)}, \quad a=\frac{2 \pi \hbar^{2} n_{t}}{m T}=\frac{T_{0}}{T}, \\
T_{c}^{L}=\frac{\pi \hbar^{2} n_{t}}{2 m \tilde{\epsilon}^{L}}=\frac{T_{0}}{4 \tilde{\epsilon}^{L}},
\end{gathered}
$$

but also to perform a complete calculation of the VR superfluid exciton density. (In Eqs. (9) and (10) the quantity $T_{0}=2 \pi \hbar^{2} n_{t} / m$ is the degeneration temperature of the spin-polarized excitons; if the excitons are not spin-polarized and have $g_{e x}$ spin degrees, the real exciton degeneration temperature is $2 \pi \hbar^{2} n_{t} / g_{e x} m$, this quantity is in $g_{e x}$ times less than $T_{0} ; g_{e x}=4$ for GaAs.) At typical total exciton density (in all spin degrees [41]) $n_{t}=2 \cdot 10^{10}$ $\mathrm{cm}^{-2}$ and exciton mass $m=0.22 m_{0}\left(m_{0}\right.$ is free electron mass) 22] the indirect exciton superfluid crossover temperature in $2 \mathrm{D}$ flat trap is estimated as $T_{c}^{L}=1.2 \mathrm{~K}$ for the trap size $L=5.6 \mu \mathrm{m}, T_{c}^{L}=1.15 \mathrm{~K}$ for $L=43 \mu \mathrm{m}$, $T_{c}^{L}=1.1 \mathrm{~K}$ for $L=14 \mathrm{~cm}$ and $T_{c}^{L}=1.077 \mathrm{~K}$ for an infinite $(L=\infty)$ system (see Eqs. (6) and (10), degeneration temperature being equal to $T_{d e g}=1.26 \mathrm{~K}$.

In Fig. 2 we show the temperature dependence of the (VR) superfluid exciton density in 2D flat trap. The temperature unit is $\mathrm{KT}$ temperature of the $2 \mathrm{D}$ infinite superfluid [35]

$$
\left.T_{c}^{\infty} \equiv T_{c}^{L}\right|_{L=\infty}=\frac{\pi \hbar^{2} n_{t}}{2 m \tilde{\epsilon}^{\infty}}=\frac{T_{0}}{4 \tilde{\epsilon}^{\infty}},
$$

(here, as in Eq. (10), we assume $n_{l}\left(T_{c}^{\infty}\right)=n_{t}$; see Eq. (8)). We see that the superfluid density universal jump $\tilde{n}_{s}^{\infty}=2 m T_{c}^{\infty} / \pi \hbar^{2}=n_{t} / \tilde{\epsilon}^{\infty}$ is smeared out in a flat trap as compared to infinite systems. Besides smearing, the confinement affects in growth of the superfluid crossover temperature.

The dependence of superfluid crossover temperature $T_{c}^{L}$ on trap size $L$ are depicted in Fig. 1. We see the fit (6) is much more better coincides with the exact KT-model numerical intergation of Eq. (2), than the Kosterlitz's renormalization group calculation 20]

$$
\tilde{\epsilon}_{R G}^{L}=\left(1-\frac{\pi^{2} b^{2}}{\ln ^{2}\left(L / r_{0}\right)}\right) \tilde{\epsilon}^{\infty}
$$

(although, in the strict sence, Eqs. (6) and (12) coincide in the logarithmic approximation).

\section{SUPERFLUIDITY OF 2D EXCITONS IN A LARGE-SIZE HARMONIC TRAP: THE GENERALIZED LOCAL DENSITY APPROXIMATION}

The local density approximation for trapped excitons described in Sect. Пis valid only if the total exciton density varies sufficiently slowly on both typical phonon and vortex scales of the trapped superfluid. But the typical vortex scales of $2 \mathrm{D}$ homogeneous infinite superfluid have the divergence near KT transition [20, 42]. So, close to KT transition the vortex scales cannot be much smaller than the (finite) trap size (TF radius) $\bar{L}$. Moreover, at sufficiently low temperatures the density of (thermally created) vortex pairs is negligible throughout the trap except the narrow region near the boundary of the system. Thus, for more accurate description of the exciton superfluidity in 2D inhomogeneous traps the usual LDA must be modified.

The generalization of KT rare vortex pair gas theory for inhomogeneous systems is not a simple problem. Indeed, vortex charge $q$ being proportional to square $\operatorname{root} \sqrt{n_{l}(\mathbf{R})}$ of VU superfluid exciton density $n_{l}(\mathbf{R})[19]$ 
varies as trap point $\mathbf{R}$ displaces. So, vortex pairs are no longer neutral dipoles. This result in an inapplicability, in the strict sense, of KT theory for a inhomogeneous case. However, this theory describes correctly the superfluid on the small length scales (much smaller than trap size $\bar{L}$ ), on which the vortex charge varies slowly. On the scales of order of $\bar{L}$ the integration of Eq. (2) must be effectively cutted off. But as in a flat trap, in a harmonic trap vortex properties depend logarithmically on the length scale. So, at sufficiently large $\bar{L}$ $\left(\ln \left(\bar{L} / r_{0}\right) \gg 1\right)$ in the logarithmic approximation the exact value of the cutoff in integrating Eq. (2) is not impotant. This results in appearing a possibility to investigate quantitatively CQW exciton superfluid in a largesize harmonic trap with a logarithmically small error in the approximation which we will call generalized local density approximation (GLDA) for KT theory of weakly inhomogeneous systems. In GLDA, VR exciton superfluid properties in 2D large-size harmonic trap near to trap point $\mathbf{R}$ are replaced by ones in $2 \mathrm{D}$ flat trap with total density $n_{t}$ equal to total density $n_{t}(\mathbf{R})$ in harmonic trap, flat trap size $L$ being of order of harmonic trap size $\bar{L}$.

We obtain readily the exciton fluid in 2D symmetrical harmonic trap at low temperatures to divide in GLDA, as in LDA, into the superfluid circle

$$
n_{t}(R)>\tilde{n}_{t}^{L}, \quad n_{s}(R) \sim n_{t}(R) \quad\left(R<R_{s}^{L}\right)
$$

and normal ring

$$
n_{t}(R)<\tilde{n}_{t}^{L}, \quad n_{s}(R) \approx 0 \quad\left(R_{s}^{L}<R<\bar{L}\right) .
$$

Here $R_{s}^{L}$ is the superfluid circle radius in the harmonic trap of size $\bar{L}$, and (see Eq. (10))

$$
\tilde{n}_{t}^{L}=\frac{2 m \tilde{\epsilon}^{L}}{\pi \hbar^{2}} T
$$

is (critical) total exciton density in 2D flat trap of size $L \sim \bar{L}$, at which excitons undergo the superfluid crossover at temperature $T$.

But near the circumference $R \approx R_{s}$, where $n_{t}(R) \approx$ $\tilde{n}_{t}^{L}$, which separates the superfluid circle and normal ring, there is a narrow region of the local KT crossover, on which the onset of the pair vortex dissociation and free vortex formation is gradual. This is one of distinctions between LDA and GLDA.

The critical total density (15) grows with the exciton temperature. So, superfluid circle radius $R_{s}^{L}$ drops, the superfluid circle shrinks, and the normal ring becomes wider (see Eqs. (13)-(15)). At the temperature (see Eq. (15))

$$
\bar{T}_{c}^{L}=\frac{\pi \hbar^{2} n_{t}^{m}}{2 m \tilde{\epsilon}^{L}}
$$

at which the critical total exciton density $\tilde{n}_{t}^{L}$ is equal to the maximal one in whole the trap $n_{t}^{m}=n_{t}(0)$ the excitons undergoes the global superfluid crossover where
VR superfluid component vanishes (in contrast to LDA) gradually. Above $\bar{T}_{c}^{L}$ there is no superfluid circle, so the trapped excitons are in globally normal phase.

We describe quantitatively the superfluidity of $2 \mathrm{D}$ CQW excitons in a large-size harmonic trap in GLDA for TF density profile [6, 38]

$$
n_{t}(R)=n_{l}(R)=n_{t}^{m}\left(1-R^{2} / \bar{L}^{2}\right) \theta(\bar{L}-R),
$$

where $\theta(x)=0$ if $x<0$ and $\theta(x)=1$ if $x>0$. In this case, we obtain in GLDA the following equations for superfluid circle radius $R_{s}^{L}$, global superfluid crossover temperature $\bar{T}_{c}^{L}$, TF radius $\bar{L}$, exciton chemical potential $\mu$, maximal total exciton density $n_{t}^{m}$ (in trap center), and exciton numbers in the superfluid circle $N_{S C}$ and normal $\operatorname{ring} N_{N R}$ :

$$
\begin{gathered}
R_{s}^{L}=\bar{L} \sqrt{1-\tilde{n}_{t}^{L} / n_{t}^{m}}=\bar{L} \sqrt{1-T / \bar{T}_{c}^{L}}, \\
\bar{T}_{c}^{L}=\frac{\hbar \omega_{o} \sqrt{N_{t}}}{2 \tilde{\epsilon}^{L} \sqrt{2 \zeta^{\prime}}} \\
\bar{L}=x_{o} \sqrt[4]{8 \zeta^{\prime} N_{t}}, \\
\mu=\hbar \omega_{o} \sqrt{2 \zeta^{\prime} N_{t}}, \\
n_{t}^{m}=\frac{\sqrt{N_{t} / 2 \zeta^{\prime}}}{\pi x_{o}^{2}}, \\
N_{S C}=N_{t}\left(1-\left(T / \bar{T}_{c}^{L}\right)^{2}\right) \theta\left(\bar{T}_{c}^{L}-T\right), \\
N_{N R}=N_{t}\left(\left(T / \bar{T}_{c}^{L}\right)^{2} \theta\left(\bar{T}_{c}^{L}-T\right)+\theta\left(T-\bar{T}_{c}^{L}\right)\right),
\end{gathered}
$$

where $\omega_{o}$ is the oscillator (trap) frequency, $x_{o}=\sqrt{\hbar / m \omega_{o}}$ is the oscillator length, $N_{t}$ is the total exciton number in the trap (in all spin degrees [41]), and $\zeta^{\prime}=$ $\mu m / 2 \pi \hbar^{2} n_{t}^{m}=$ const is dimensionless exciton chemical potential, which we assume to be independent of $T$ and $n_{t}^{m}$. Local and global superfluid crossover halfwidths determining the correctness of the logarithmic approximation (and hence, GLDA), as in a flat trap, have the third order on the logaritmically small quantity, i. e.,

$$
\begin{gathered}
\Delta R_{s}^{L} \sim \frac{\bar{L}^{2} T}{2 R_{s}^{L} \bar{T}_{c}^{L}} \frac{\pi^{2} b^{2}}{\left(\ln \left(\bar{L} / r_{0}\right)+\Delta\right)^{3}}, \\
\Delta \bar{T}_{c}^{L} \sim \bar{T}_{c}^{L} \frac{\pi^{2} b^{2}}{\left(\ln \left(\bar{L} / r_{0}\right)+\Delta\right)^{3}} .
\end{gathered}
$$

In Fig. 3 we show (VR) superfluid profiles for 2D harmonically trapped CQW excitons in GLDA and in LDA (see Fig. 3(a)) at different temperatures below $\bar{T}_{c}^{L}$ (the 

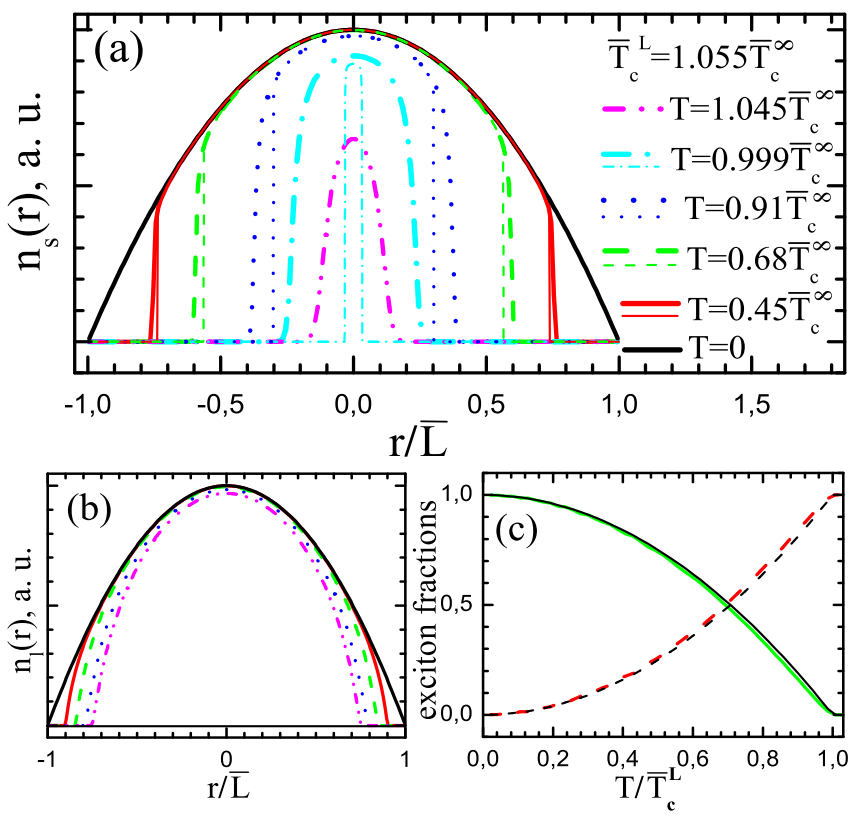

FIG. 3: (a) The vortex-renormalized superfluid profiles of 2D CQW excitons in the harmonic trap of large radius $\bar{L}$ $\left(\ln \left(\bar{L} / r_{0}\right)=8\right)$ at different temperatures $T$ in GLDA (thick lines) and in LDA (thin lines). (b) The vortex-unrenormalized superfluid profiles in CQW structure (studied experimentally in Ref. [12, 22] ) for $n_{t}^{m}=2.5 \cdot 10^{10} \mathrm{~cm}^{-2}$ at the same temperatures for corresponding lines as in (a). (c) Solid lines shows the temperature dependence of superfluid (thick) and superfluid circle (thin) fractions. Dash lines denotes normal (thick) and normal ring (thin) fractions.

temperature unit is the global superfluid transition temperature in LDA $\left.\bar{T}_{c}^{\infty} \equiv \bar{T}_{c}^{L}\right|_{L=\infty}=\left(\tilde{\epsilon}^{L} / \tilde{\epsilon}^{\infty}\right) \bar{T}_{c}^{L}<\bar{T}_{c}^{L}$; see Eqs. (6), (10), (11) and (16)). VU superfluid profiles are presented in Fig. 3(b) for CQW structure (see, e. g., [12, 22]) with exciton mass $m=0.22 m_{0}$, dielectric constant $\varepsilon=12.5$, and effective electron-hole layer distance $d=13.6 \mathrm{~nm}$ for trap center total exciton density being equal to $n_{t}^{m}=2.5 \cdot 10^{10} \mathrm{~cm}^{-2}$ [38]. One see the superfluid density on the superfluid circle boundary region to drop sharply far from the global superfluid transition
$\left(T \not \bar{T}_{c}^{L}\right)$. Besides, there is little normal component in the superfluid circle at $T \not \bar{T}_{c}^{L}$ (for a pure CQW structure). The latter fact is visually depicted in Fig. 3(c), where the curve of the superfluid (normal) exciton number in GLDA quite merge with that of exciton number in the superfluid circle (normal ring).

Because the global superfluid crossover temperature $\bar{T}_{c}^{L}$ for the harmonic trap of radius $\bar{L}$ coincides with the temperature $T_{c}^{L}$ for the flat trap of size $L \sim \bar{L}$ (see above), the dependence of the former on $\bar{L}$ coincides with that presented in Fig. 1 with the change $T_{c}^{L} \rightarrow \bar{T}_{c}^{L}$, $T_{c}^{\infty} \rightarrow \bar{T}_{c}^{\infty}$, and $L \rightarrow \bar{L}$.

\section{CONCLUSION}

We have calculated the temperature dependence of superfluid density and the superfluid crossover temperature for 2D spatially indirect CQW excitons in a large-size flat trap as well as superfluid profiles and global superfluid crossover temperature for the excitons in a large-size harmonic trap. We reveal that harmonically trapped exciton system at low temperatures is divided into the superfluid circle (with superfluid component) and normal ring (without it). At their boundary there is the narrow crossover region at which the onset of vortex pair dissociation and free vortex formation takes place. The superfluid density goes rather sharply to zero in this region. As exciton temperature grows superfluid circle shrinkes to zero. This temperature corresponds to the global superfluid transition (crossover) in the harmonic trap. At this temperature the Bose condensate also disappears (details will be published elsewhere).

\section{Acknowledgements}

Authors are thankful to P. Littlewood, L. V. Keldysh, R. Zimmermann, and D. Snoke for useful discussions of the results. The work was supported by the Russian Foundation of Basic Research and Swedish Foundation for Strategic Research (SSR).
[1] Yu.E. Lozovik, V.I. Yudson, JETP Lett. 22 (1975) 274; JETP 44 (1976) 389; Solid State Commun. 19 (1976) 391; ibid. 21 (1977) 211; Yu.E. Lozovik, V.N. Nishanov, Phys. Solid State 18 (1976) 1905; Yu.E. Lozovik, A.M. Ruvinsky, JETP 85 (1997) 979; Yu.E. Lozovik, Report on Conf. Diel. Electr., FAN, 1973.

[2] A. Filinov, P. Ludwig, Yu. E. Lozovik, M. Bonitz, H. Stolz, J. Phys: Conf. Series 35 (2006) 197; P. Ludwig, A. Filinov, M. Bonitz, H. Stolz, Phys. Status Solidi B 243 (2006) 2363.

[3] O.L. Berman, Yu.E. Lozovik, D.W. Snoke, R.D. Coalson, Phys. Rev. B 70 (2004) 235310; ibid. 73 (2006) 235352; Solid State Commun. 134 (2005) 47; Physica E 34 (2006) 268; J. Phys.: Condens. Matter 19 (2007) 386219.
[4] Yu.E. Lozovik, O.L. Berman, JETP Lett. 64 (1996) 573; JETP 84 (1997) 1027; Phys. Scripta 55 (1997) 491; Yu.E. Lozovik, O.L. Berman, V.G. Tsvetus, ibid. 66 (1997) 355; Phys. Rev. B 59 (1999) 5627; Yu.E. Lozovik, O.L. Berman, M. Willander, J. Phys.: Condens. Matter 14 (2002) 12457; Yu. E. Lozovik, S. A. Verzakov, M. Willander, Phys. Lett. A 260 (1999) 400; Yu.E. Lozovik, M. Willander, Appl. Phys. A 71 (2000) 379.

[5] G. Vignale, A.H. MacDonald, Phys. Rev. Lett. 76 (1996) 2786; X. Zhu, P.B. Littlewood, M.S. Hibertsen, T.M. Rice, ibid. 74 (1995) 1633; Y. Naveh, B. Laikhtman, ibid. 77 (1996) 900; J. Fernández-Rossier, C. Tejedor, ibid. 78 (1997) 4809; A.V. Balatsky, Y.N. Joglekar, P.B. Littlewood, ibid. 93 (2004) 266801; Y.N. Joglekar, A.V. 
Balatsky, M.P. Lilly, Phys. Rev. B 72 (2005) 205313; S. Conti, G. Vignale, A.H. MacDonald, ibid. 57 (1998) 6846; J.F. Jan, Y.C. Lee, ibid. 58 (1998) 1714; A.L. Ivanov, P.B. Littlewood, H. Haug, ibid. 59 (1999) 5032; S.-R.E. Yang, J. Yeo, S. Han, ibid. 71 (2005) 245307; A.L. Ivanov, Europhys. Lett. 59 (2002) 586.

[6] Yu.E. Lozovik, S.Yu. Volkov, M. Willander, JETP Lett. 79 (2004) 473; G.E. Astrakharchik et al., arXiv:0707.4630.

[7] Yu.E. Lozovik, O.L. Berman, Phys. Scripta 58 (1998) 86; Phys. Solid State 40 (1998) 1228; D.V. Kulakovsky, Yu.E. Lozovik, A.V. Chaplik, JETP 99 (2004) 850.

[8] G.E. Astrakharchik et al., Phys. Rev. Lett. 98 (2007) 060405; H.P. Büchler et al., ibid. 98 (2007) 060404; S. De Palo, F. Rapisarda, G. Senatore, ibid. 88 (2002) 206401; Yu.E. Lozovik, E.A. Rakoch, Phys. Lett. A 235 (1997) 55; A.I. Belousov, Yu.E. Lozovik, JETP Lett. 68 (1998) 858; Eur. Phys. J. D 8 (2000) 251; A. Filinov, M. Bonitz et al., Phys. Status Solidi C 3 (2006) 2457; C. Mora, O. Parcollet, X. Waintal, Phys. Rev. B 76 (2007) 064511.

[9] Yu.E. Lozovik, V.I. Yudson, Solid State Commun. 22 (1977) 117; A.V. Klyuchnik, Yu.E. Lozovik, JETP 49 (1979) 335; J. Phys. C 11 (1978) L483; S.I. Shevchenko, Phys. Rev. Lett. 72 (1994) 3242; Yu.E. Lozovik, A.V. Poushnov, Phys. Lett. A 228 (1997) 399.

[10] B. Laikhtman, Europhys. Lett. 43 (1998) 53; P. Stenius, Phys. Rev. B 60 (1999) 14072; Yu.E. Lozovik, I.V. Ovchinnikov, ibid. 66 (2002) 075124; JETP Lett. 74 (2001) 288; Solid State Commun. 118 (2001) 251; Yu.E. Lozovik, I.L. Kurbakov, I.V. Ovchinnikov, ibid. 126 (2003) 269; R. Zimmermann ibid. 134 (2005) 43; A. Olaya-Castro, F.J. Rodríguez, L. Quiroga, C. Tejedor, Phys. Rev. Lett. 87 (2001) 246403; J. Keeling, L.S. Levitov, P.B. Littlewood, ibid. 92 (2004) 176402; L.S. Levitov, B.D. Simons, L.V. Butov, ibid. 94 (2005) 176404.

[11] S.A. Moskalenko, D.W. Snoke, Bose-Einstein condensation of excitons and biexcitons and coherent nonlinear optics with excitons, Cambridge University Press, Cambridge, 2000.

[12] V.B. Timofeev, Phys.-Uspekhi 48 (2005) 295; V.B. Timofeev, A.V. Gorbunov, A.V. Larionov, J. Phys.: Condens. Matter 19 (2007) 295209.

[13] L.V. Butov, J. Phys.: Condens. Matter 16 (2004) R1577; ibid. 19 (2007) 295202.

[14] D. Snoke, Science 298 (2002) 1368; R. Rapaport, G. Chen, J. Phys.: Condens. Matter 19 (2007) 295207; Z. Vörös et al., ibid. 19 (2007) 295216.

[15] L.V. Keldysh, Y.V. Kopaev, Phys. Solid State 6 (1965) 2219; A.N. Kozlov, L.A. Maksimov, JETP 21 (1965) 790; L.V. Keldysh, A.N. Kozlov, ibid. 27 (1968) 521.

[16] P.C. Hohenberg, Phys. Rev. 158 (1967) 383; N.D. Mermin, H. Wagner, Phys. Rev. Lett. 17 (1966) 1133.

[17] J.W. Kane, L.P. Kadanoff, Phys. Rev. 155 (1967) 80.

[18] V.L. Berezinskii, JETP 32 (1970) 493; ibid. 34 (1971) 610.

[19] J.M. Kosterlitz, D.J. Thouless, J. Phys. C 6 (1973) 1181.

[20] J.M. Kosterlitz, J. Phys. C 7 (1974) 1046.
[21] V. Bagnato, D. Kleppner, Phys. Rev. A 44 (1991) 7439; W. Ketterle, N.J. van Druten, ibid. 54 (1996) 656.

[22] A.V. Gorbunov, V.E. Bisti, V.B. Timofeev, JETP 101 (2005) 693; A.V. Larionov, V.B. Timofeev el al., ibid. 90 (2000) 1093; JETP Lett. 71 (2000) 117; ibid. 75 (2002) 200; ibid. 75 (2002) 570; A.V. Larionov, V.B. Timofeev, ibid. 73 (2001) 301; A.A. Dremin, V.B. Timofeev, A.V. Larionov et al., ibid. 76 (2002) 450; A.V. Gorbunov, V.B. Timofeev, ibid. 83 (2006) 146; ibid. 84 (2006) 329; A.V. Gorbunov, A.V. Larionov, V.B. Timofeev, ibid. 86 (2007) 46; V.B. Timofeev, A.V. Gorbunov, J. Appl. Phys. 101 (2007) 081708; A.A. Dremin, A.V. Larionov, V.B. Timofeev, Phys. Solid State 46 (2004) 170.

[23] L.V. Butov et al., Nature (London) 417 (2002) 47.

[24] V.V. Krivolapchuk, E.S. Moskalenko, A.L. Zhmodikov, Phys. Rev. B 64 (2001) 045313.

[25] A.T. Hammack et al., Phys. Rev. Lett. 96 (2006) 227402.

[26] Z. Vörös et al., Phys. Rev. Lett. 97 (2006) 016803.

[27] D.W. Snoke et al., Solid State Commun. 134 (2005) 37.

[28] A.T. Hammack et al., J. Appl. Phys. 99 (2006) 066104.

[29] M. Willander, O. Nur, Yu.E. Lozovik et al., Microelectron. J. 36 (2005) 940.

[30] G. Chen et al., Phys. Rev. B 74 (2006) 045309.

[31] D. Snoke et al., Nature (London) 418 (2002) 754; Solid State Commun. 127 (2003) 187; R. Rapaport, G. Chen, D. Snoke et al., Phys. Rev. Lett. 92 (2004) 117405.

[32] V.V. Krivolapchuk, E.S. Moskalenko, A.L. Zhmodikov et al., Solid State Commun. 111 (1999) 49; Phys. Solid State 41 (1999) 291.

[33] L.V. Butov et al., Nature (London) 418 (2002) 751; Phys. Rev. B 58 (1998) 1980; ibid. 59 (1999) 1625; Phys. Rev. Lett. 73 (1994) 304; ibid. 86 (2001) 5608; ibid. 92 (2004) 117404; S. Yang et al, ibid. 97 (2006) 187402; C.W. Lai et al., Science 303 (2004) 503.

[34] LDA for KT theory was also discussed in talk of Yu.E.L. on the Cambridge Workshop on exciton BEC, 2004.

[35] D.R. Nelson, J.M. Kosterlitz, Phys. Rev. Lett. 39 (1977) 1201.

[36] P. Minnhagen, Phys. Rev. B 32 (1985) 3088.

[37] P. Minnhagen and G.G. Warren, Phys. Rev. B 24 (1981) 2526; P. Minnhagen, Rev. Mod. Phys. 59 (1987) 1001.

[38] Yu.E. Lozovik et al., JETP, in press (2008); Solid. State Commun. 144 (2007) 399.

[39] Yu.E. Lozovik, I.L. Kurbakov, M. Willander (to be publ.).

[40] The condition $\tilde{\epsilon}^{L}>\tilde{\epsilon}^{\infty} / 1.2$ corresponds to sizes $L$, obeyed the inequality $\ln \left(L / r_{0}\right)>3.2$ (see Fig. 1 and Eq. (6) $)$, i. e., it takes place for the flat traps with more than $\left(L / r_{0}\right)^{2} \sim 600$ excitons.

[41] Throughout this Letter all densities and particle numbers are given in all spin degrees. We make this denotion because it is the density in all spin components that determines VR superfluid effects. This is associated with that a vortex rotates all $g_{e x}$ spin components.

[42] V. Ambegaokar, B.I. Halperin, D.R. Nelson, E.D. Siggia, Phys. Rev. Lett. 40 (1978) 783. 